\title{
Ascitis quilosa después de nefrectomia radical laparoscópica. Tratamiento conservador sin paracentesis
}

\author{
Luis A. Fariña, María Concepción Martínez \\ Servicios de Urología y Radiología. Hospital Povisa. Vigo, Pontevedra, España.
}

\begin{abstract}
Resumen
Introducción: La ascitis quilosa es una complicación rara después de diferentes intervenciones abdominales y retroperitoneales. Aunque suele desaparecer con medidas conservadoras, puede ser necesaria una reintervención para sellar los vasos linfáticos que permanecen abiertos en el lecho operatorio.

Caso clínico: Una mujer de 60 años fue intervenida de nefrectomia radical laparoscópica izquierda de un tumor de 9,5 x 7,5 $\mathrm{cm}$, estadio pT2 pNO. En la intervención se hizo una disección cuidadosa del hilio, se ligaron o coagularon algunos gruesos vasos linfáticos peritumorales y se clipó el uréter distal. Unos dias después del alta, consulta por aumento del perimetro abdominal y en los estudios de imagen se encuentra ascitis quilosa difusa. Fue tratada de manera conservadora, sin realizar paracentesis diagnóstica o evacuadora, con dieta baja en sal y lípidos, y espironolactona, con curación completa en el plazo de un mes.

Comentario: Un tratamiento conservador con diuréticos, dieta baja en lípidos y eventualmente con paracentesis debe ser la primera opción de tratamiento de las ascitis quilosa después de nefrectomia. En los últimos años se han descrito técnicas laparoscópicas para resolver una ascitis quilosa de forma poco invasiva, aunque no exenta de dificultad, tratándose de una re-intervención.
\end{abstract}

Palabras clave: Ascitis quilosa. Laparoscopia. Cáncer renal.

\section{Chylous ascites following radical nephrectomy. Conservative Management whithout paracentese}

\section{Abstract}

Introduction: Chylous ascites is a rare complication after a number of abdominal and retroperitoneal surgeries. Althought conservative treatment may be curative, a reoperation to sell out the open megalymphatics in the operative field may be needed.

Patient and method: A 60 year-old woman was treated with a laparoscopic radical nephrectomy for a $9.5 \times 7.5 \mathrm{~cm}$, pT2 pNO tumor. A carefull dissection of the renal hilium was performed, distal ureter was clipped and several gross peritumoral lymphatic vessels were clipped or coagulated, with no inmediate complications. Several days after being discharged home, she complained of increased abdominal perimeter and on image studies a difuse chylous ascitis was found. She was treated in a conservative way, with no diagnostic or palliative paracentesis, low salt and lipids diet, and espironolactone, with complete cure in a month. After six months of follow-up, no simptoms nor CT- signs of ascites or tumor recurred.

Comment: A conservative treatment, with diuretics, low lipids and salt diet, and eventually repeated paracentesis should be the first options for chylous ascites after nephrectomy. In the last years, several laparoscopic techniques had been described to treat chylous ascites in a low invasive way, but the intrinsic difficulties of such a re-operation much be considered.

$\mathrm{L}^{2}$ a ascitis quilosa, que es el acúmulo intraperitoneal del líquido rico en triglicéridos procedente de la linfa intestinal, es casi siempre secundaria a neoplasia o infección intraabdominal, o bien una complicación rara consecuencia de lesión operatoria de los vasos linfáticos de mayor calibre en el curso de diferentes intervenciones retroperitoneales o de la vecindad de la raiz del mesenterio. En el área urologica, en particular, se relaciona con la nefrectomia radical, la nefrectomia del donante renal y la linfadenectomia retro- peritoneal de tumor de teste ${ }^{1-4}$. Aunque suele desaparecer con medidas conservadoras, entre las que se pueden incluir la administración de diuréticos, de somatostatina o de su derivado sintético octreótida, dietas especiales bajas en sal y grasas y la parecentesis, en ocasiones es refractaria a estas medidas y puede ser necesaria una intervención para sellar los vasos linfáticos que permanecen abiertos en el lecho operatorio, o una derivación peritoneo-venosa, resultando en una morbilidad y mortalidad significativas ${ }^{5}$. 
Describimos un caso de esta infrecuente complicación después de nefrectomia radical laparoscópica, destacando que un tratamiento conservador puede ser exitoso, permitiendo eludir otras opciones que tienen riesgo de complicaciones, incluyendo la paracentesis repetida.

\section{CASO CLÍNICO}

Una mujer de 60 años fue intervenida de nefrectomía radical laparoscópica izquierda de un tumor renal del polo inferior, de 9,7x7,5 cm. En la intervención se encontraron adherencias peritumorales paraaórticas y al mesocolon, donde se ligaron o coagularon algunos vasos linfáticos gruesos, se hizo una disección cuidadosa del hilio y se clipó el uréter distal, sin incidencias intraoperatorias y con un sangrado menor de $100 \mathrm{ml}$. El estudio anatomopatológico demostró carcinoma renal de células claras, sólido y quístico, con áreas de sangrado intratumoral, grado 2-3 de Fuhrman, estadio pT2 pNO. El postoperatorio cursó sin incidencias, se detectó un descenso de la hemoglobina de 1,5 g/dl (de 13,2 a 11,7), y fue alta hospitalaria a los cinco días.

Un mes después del alta, consulta por aumento del perimetro abdominal que le causa disnea y disconfort y en los estudios de imagen se encuentra ascitis de distribución difusa, sugestiva de ser ascitis quilosa (Fig. 1). Los análisis mostraron $\mathrm{Hb}$ de $11,1 \mathrm{~g} / \mathrm{dl}$, fórmula leucocitaria y recuento de linfocitos normal, función renal y hepática normal e hipo-proteinemia leve -albúmina de 2,9 g/dl (N: 3,45), proteinas totales de 5,8 g/dl (N: 6,4-8,2). Fue tratada de manera conservadora, sin realizar paracentesis diagnóstica o evacuadora, con dieta baja en sal y lípidos, suplementos de proteinas y espironolactona $50 \mathrm{mg} /$ día, con mejoria sintomática y del perímetro abdominal, alta hospitalaria a los seis dias, y con curación completa comprobada mediante ecografía de abdomen un mes después. Seis meses después no tiene síntomas ni signos de recidiva, y la ecografía abdominal es normal.

\section{COMENTARIO}

Aún siendo una complicación rara de la cirugía urológica retroperitoneal, en fechas recientes se han comunicado un número inusual de ascitis quilosa, sobre todo después de la nefrectomía laparoscópica del donante renal y la linfadenectomía retroperitoneal laparoscópica de tumor de teste ${ }^{4,6-10}$. Aunque hay una magnificación del campo quirúrgico, el aumento de la presión proporcionada por el neumoperitoneo podría dificultar la identificación efectiva de algunos linfáticos de mayor calibre, que deben ser clipados o coagulados para lograr una linfostasis completa ${ }^{3}$.

El tratamiento de la ascitis quilosa debe establecerse de manera secuencial, de menor a mayor morbilidad. Un tratamiento conservador, con diuréticos, reposición de albúmina, dieta baja en lípidos y eventualmente con paracentesis debe ser la primera

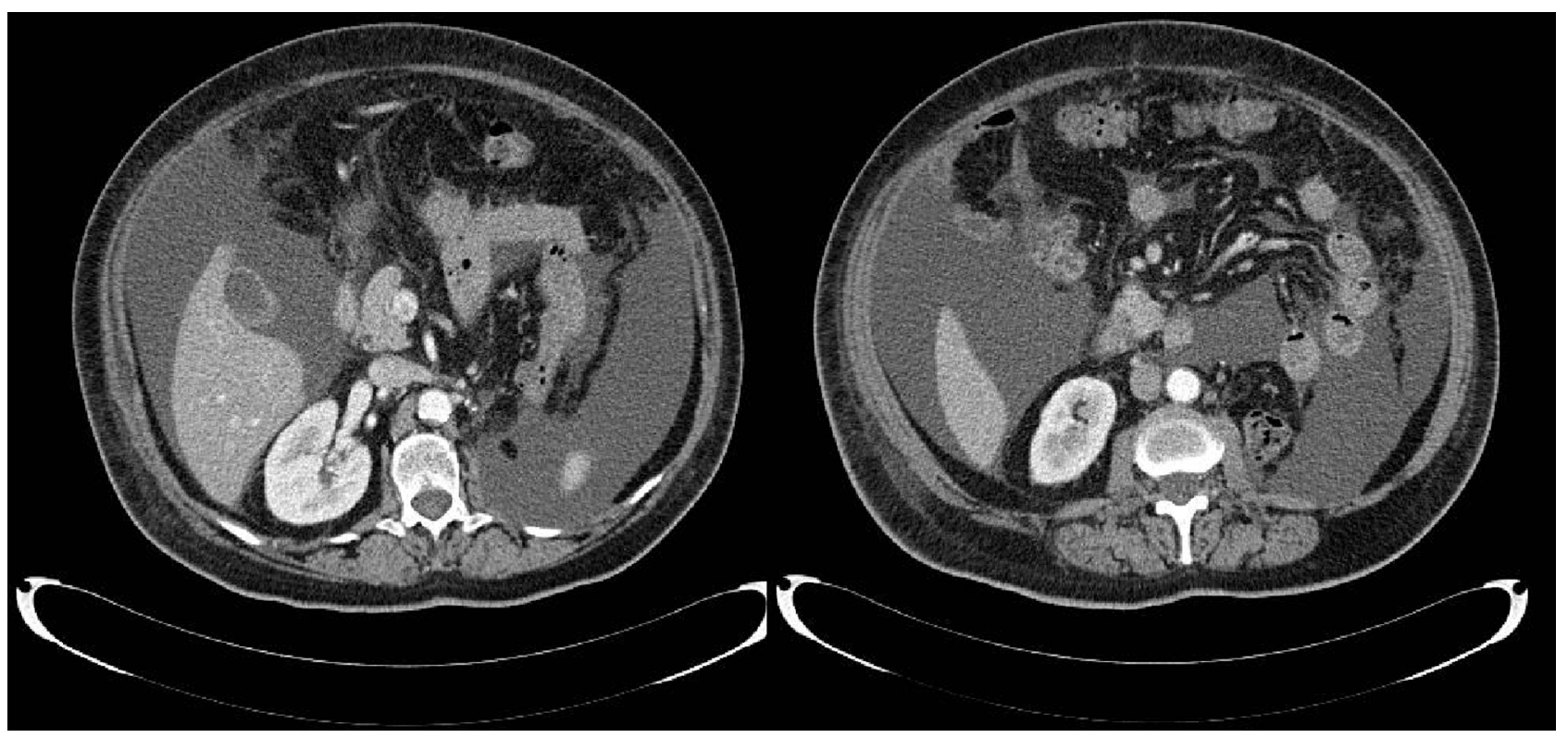

FIGURA 1. TAC de abdomen postoperatorio, secciones a nivel del hilio renal y del polo renal inferior. Se observa ascitis difusa, con líquido perihepático, parietocólico y entre las hojas del mesenterio, lo que es típico de la ascitis benigna. 
opción de tratamiento. Las dietas especiales con lipidos de absorción temprana, el uso de la somatostatina o la octreótida y la nutrición parenteral total, son la segunda opción antes de la reintervención quirugica ${ }^{5}$. La indicación juiciosa de estas opciones debe basarse en la sintomatología, y en datos clínicos como el estado nutricional y el volumen de las pérdidas, en el paciente con paracentesis repetidas. Algunos han propuesto un tratamiento máximo de forma precoz, -incluso considerando una intervención quirúrgica temprana, si el paciente tiene un riesgo quirúrgico bajo-, pues en ocasiones son necesarias semanas o meses para lograr una resolución eficaz de la ascitis por medios conservadores. En nuestra paciente, sin embargo, tuvo éxito una pauta aún más conservadora que la recomendada en los algoritmos de tratamiento más recientes.

En los últimos años se han descrito algunos casos de ascitis quilosa resueltos mediante técnica laparoscópica, de manera poco invasiva, aunque no exenta de dificultad, por tratarse de una reintervención $^{8,11-13}$, y no siempre exitosa ${ }^{14}$. En estas técnicas, es necesario utilizar un colorante lipofílico ingerido inmediatamente antes de la intervención, o bien una dieta rica en grasas, con objeto de aumentar la posibilidad de identificar el o los megalinfáticos abiertos. Si no se identifica claramente el origen de la ascitis, se recomienda la ligadura o clipado de todos los linfaticos del área sospechosa de fístula usando clips o pegamentos biocompatibles.

\section{REFERENCIAS}

1. Isa Kroon WA, Sánchez de la Muela P, Ucar Terren A, Robles García JE, Zudaire Bergera JJ, Berián Polo JM. Linforragia postlinfadenectomia por adenocarcinoma renal. Actas Urol Esp. 1988;12(4):371-373.

2. Santos D, Páez A, Valer MP, Luján M, Pascual C, Berenguer A. Ascitis quilosa como complicación de una linfadenectomia retroperitoneal. Gastroenterol Hepatol. 2004;27:150.
3. Negoro H, Oka H, Kawakita M. Two cases of chyloretroperitoneum following retroperitoneoscopic nephroureterectomy. Int J Urol. 2006;13(4):487-489.

4. Evans JG, Spiess PE, Kamat AM, Wood CG, Hernandez M, Pettaway CA, et al. Chylous ascites after post-chemotherapy retroperitoneal lymph node dissection: review of the M. D. Anderson experience. J Urol. 2006;176(4 Pt 1):1463-1467.

5. Leibovitch I, Mor Y, Golomb J, Ramon J. The diagnosis and management of postoperative chylous ascites. J Urol. 2002;167 (2 Pt 1):449-457.

6. Bachmann A, Ruszat R, Dickenmann M, Giannini O, Mayr M, Steiger J, et al. Chyloretroperitoneum with secondary chylothorax after retroperitoneoscopic donor nephrectomy. Urology. 2005;66(4):881.

7. Caumartin Y, Pouliot F, Sabbagh R, Dujardin T. Chylous ascites as a complication of laparoscopic donor nephrectomy. Transpl Int. 2005;18(12):1378-1381.

8. Molina WR, Desai MM, Gill IS. Laparoscopic management of chylous ascites after donor nephrectomy. J Urol. 2003;170(5): 1938.

9. Shafizadeh SF, Daily PP, Baliga P, Rogers J, Baillie GM, Rajagopolan PR. Chylous ascites secondary to laparoscopic donor nephrectomy. Urology. 2002;60(2):345.

10. Sharma A, Heer M, Subramanaym Malladi SV, Minz M. Chylous ascites after laparoscopic donor nephrectomy. J Endourol. 2005;19(7):839-840.

11. Castillo OA, Litvak JP, Kerkebe M, Olivares R, Urena R. Case report: laparoscopic management of massive chylous ascites after salvage laparoscopic retroperitoneal lymph-node dissection. J Endourol. 2006;20(6):394-396.

12. Geary B, Wade B, Wollmann W, El-Galley R. Laparoscopic repair of chylous ascites. J Urol. 2004;171(3):1231-1232.

13. Nishizawa K, Ito $\mathrm{N}$, Yamamoto $\mathrm{S}$, Kamoto $\mathrm{T}$, Ogawa O. Successful laparoscopic management of chylous ascites following laparoscopic radical nephrectomy. Int J Urol. 2006;13(5): 619-621.

14. Link RE, Amin N, Kavoussi LR. Chylous ascites following retroperitoneal lymphadenectomy for testes cancer. Nat Clin Pract Urol. 2006;3(4):226-232.

Correspondencia autor: Dr. Luis A. Fariña Pérez

Servicio de Urologia. Hospital Povisa

Salamanca, 5, 36211 Vigo, Pontevedra

Tel.: 986413144

E-mail autor: luisfarina@yahoo.com

Información artículo: Nota clínica

Trabajo recibido: octubre 2007

Trabajo aceptado: noviembre 2007 